\title{
Attitude Calibration Technology for Radar Antenna Based on Airborne
}

\author{
HE Hongli ${ }^{1, a}$, HU Shaolin ${ }^{2, b}$ \\ ${ }^{1}$ Chinese Flight Test Establishment, Xi'an 710089, China \\ ${ }^{2}$ Key Laboratory of Spacecraft Faults Diagnosis and Maintance, Xi'an, 710043, China \\ ahhhl2003@163.com, ${ }^{b}$ hfkth@126.com
}

Keywords:Radar antenna; flight test ; attitud; calibration ; error analysis

\begin{abstract}
After ending the Radar Antenna modification on the airborne plane, the initial installation error is need to be measured and to be compensated with the initial error. For the attitude measuring requirements of airborne radar Antenna mounted, This paper studies a non-contact optical measuring method based on the total station(a laser measurement equipment), which can solve the high precise attitude measuring problem. At first multiple points on the surface of Radar target and plane axis are measured, transform the corrdinate of Radar to the plane corrdinate to make the points fitting to the plane, then the points of Radar target are fitted to a plane;the attitude are obtained though the calculation and analysis from the fitting plane and the specified line,The principle ,scheme,calculating method and error analysis for airborne Radar antenna, have been detailed in the following papers. The measurement and algorithm method have been widely used in attitude measurement of weapons mounted, which are good to apply in the measurement; which can be applied in the other weapons target mounted on the airborne.
\end{abstract}

\section{Introduction $^{1}$}

The test of weapon system is an important subject for flight testWeapon system are equiped with many kinds of radar, photoelectric collimator, etc .The precision of weapon system should be authenticated comprehensively mainly by fight test in the air[1]., which checks the precision of all kinds of radar on the airplane. Radar should be consistent with the airplane's coordinates, which is equipped on the airplane with relevant requirements, so that the weapon system can build the same operation target with the airplane[2]. During mounting radar on airborne, radar should be consistent with the attitude of the airplane's coordinates as far as possible. Because of the errors of the installation between the baseline of antenna system and the airplane's heading axis, the precision of tracking and measurement with equipments will be seriously influenced without checking modification[3] [4].

In order to complete Weapon system performance test, The first technical problem is to abtain the accurate result for radar calibration. Currently, the methods for testing the attitude of installation almost are that through measuring the threer base points on the target plane of radar antenna, and then calculate the attitude through the specified line Which is based on the geometric relationship of the positioning result. But for the small target plane, there is a low precision result through the method, which cannot be satisfied with the precise requirement in measurement. This paper studies on the algorithm of the attitude for the target plane, which can improve the precision of attitude measurement. At first, the multiple points on surface of plane are measured,transform the corrdinate of radar to the plane corrdinate to make the points fit to the plane, In the end, the attitude are obtained by calculation and analysis.

\section{Target Plane Attitude Check}

\subsection{Principle of Target Plane Attitude Check[5]}

After ending the modification of the Airborne Radar target plane on the airborne plane, the angle between target plane and airplane baseline is fixed value, which can be measured by the distance

${ }^{1}$ Partly supported by the National Nature Science Fund of China (61473222) 
measurement principle. Through the relationships of the corresponding points between the target surface and base coordinates of the plane, which can be measured by total station, the computing angle between surface and surface is converted into the angle between line and line, and the angle between the target surface and the base surface of the plane can be obtained by calculation and analysis algorithm. There are some steps of measurement here. Firstly, remove the protective cover and keep the aircraft level,which are based on the specified point of the plane. measure the axis of aircraft ,calcute the error between the specified axis and the totle station axis, and set the total station axis into the measurement system of the aircraft axis.then position the device and reference points according to the layout of the aircraft target surface. For the same target surface, all points are generally measured in a measurement station. In order to keep consistent with the plane's coordinates, resection algorithm is usually used to unify the systems of coordinates. By using the adjustment algorithm, the normal vector of the target surface is obtained by adjustment process in the same coordinate system. At last, the attitude can be solved by calculation and analytical algorithm of line and line.

\subsection{Coordinate Setting[4][5]}

To carry out the measurement of the attitude, first of all, a system of coordinates should be set. Defined the system of antenna mounting platform coordinates as the system of the aircraft's body coordinates after keeping the axis horizontal .The axis Yt points to the nose of the aircraft. The axis $\mathrm{Z}$ points to the vertical . The axis $\mathrm{X}$ and the plane $\mathrm{YZ}$ constitute the system of right-handed coordinates. The origin point is defined as the origin of the system of the specified plane's coordinates, As shown in the figure 1. Antennas mounted on the aircraft's head or sides and connected with the plane tightly, as shown in the figure 2.

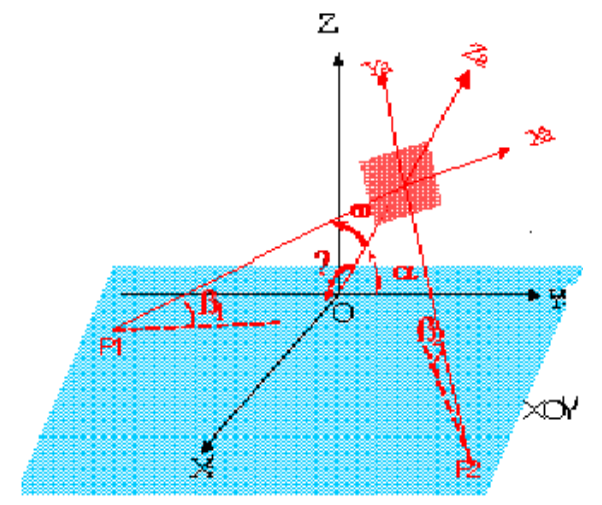

Fig1. Installation diagrammatic sketch of antenna target plane and fight

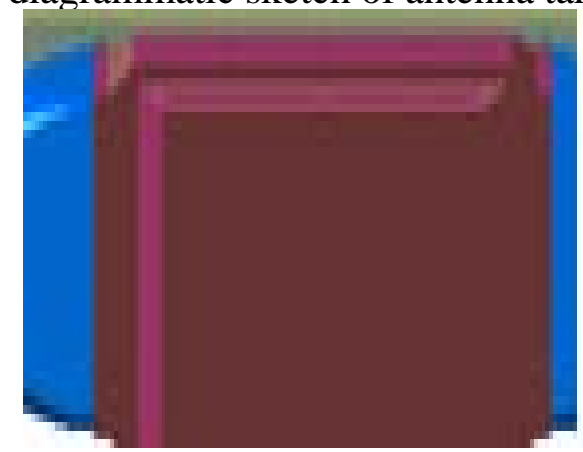

Fig 2. Radar antenna target plane mounting diagrammatic sketch

\subsection{Data Process}

Measure a number of points on the target surface and fit the plane from those measuring points. Structure the needed normal line and axis of the target surface according to the definition of the target or test requirements. Then calculated out the angle of the desired attitude according to the angle between a line and a line.one is the specified line,the other is fitting out

\subsubsection{Model}

The equation of space plane is written as:

$$
A x+B y+C z+D=0
$$

For formula (1), divided by A with both sides: 


$$
x+M y+N z+K=0
$$

Therefore, any space plane can be uniquely determined by the three parameters $\mathrm{M}, \mathrm{N}$ and $\mathrm{K}$ and the normal vector of the plane is $(1, \mathrm{M}, \mathrm{N})$. With 3 points in space, which are not collinear, a space plane can be uniquely identified. With considering the measurement error of the coordinates, generally we can calculate the fitting plane's parameters with at least 4 measurement points. we can calculate the fitting plane's parameters with more points which having been measured.

\subsubsection{Calculation of Plane Parameter using 3 Points}

When there are only three non collinear points P1, P2 and P3 in space, the three parameters $\mathrm{M}, \mathrm{N}$ and $\mathrm{K}$ of the plane can be uniquely identified. If the three points' coordinates are respectively ( $\mathrm{x} 1$, $\mathrm{Y} 1, \mathrm{z1}),(\mathrm{X} 2, \mathrm{Y} 2, \mathrm{Z} 2)$ and (X3, Y3, Z3), calculate them into the formula (2) and move the coordinate of the $\mathrm{X}$ item to the right of the equality, we can get the following equations:

$$
\left\{\begin{array}{l}
M y_{1}+N z_{1}+K=-x_{1} \\
M y_{2}+N z_{2}+K=-x_{2} \\
M y_{3}+N z_{3}+K=-x_{3}
\end{array}\right.
$$

The three parameters $\mathrm{M}, \mathrm{N}$ and $\mathrm{K}$ of the plane can be solved by the linear equations.

2.3.3 Calculation of Fitting Plane Parameter using More than 3 Measuring Points

Considering the measurement error of spatial points, the plane's fitting equation of each point can be defined as

$$
v+M y+N z+K=-x
$$

where $\mathrm{V}$ is the correction of the measurement error. The error equations of $\mathrm{n}$ measurement points such as formula (3) can be written as

$$
\left\{\begin{array}{l}
v_{1}+M y_{1}+N z_{1}+K=-x_{1} \\
v_{2}+M y_{2}+N z_{2}+K=-x_{2} \\
\cdots \cdots \\
v_{n}+M y_{n}+N z_{n}+K=-x_{n}
\end{array}\right.
$$

The formula (5) can be represented in matrix type

$$
V+A \bullet X=L
$$

$$
\text { where } L=\left(\begin{array}{l}
-x_{1} \\
-x_{2} \\
\cdots \\
-x_{n}
\end{array}\right), X=\left(\begin{array}{l}
M \\
N \\
K
\end{array}\right), A=\left(\begin{array}{lll}
y_{1} & z_{1} & 1 \\
y_{2} & z_{2} & 1 \\
\cdots \ldots . & \\
y_{n} & z_{n} & 1
\end{array}\right) \text {. }
$$

According to the least square principle, $\mathrm{X}$ can be calculated by

$$
X=\left(A^{T} A\right)^{-1} A^{T} L
$$

\subsubsection{Calculation of angle between the plane and the linear}

Through the method above, the parameters of the space plane can be calculated, where $\mathrm{P}(1, \mathrm{M}, \mathrm{N})$ is normal vector of a calculated plane. Through two points $\mathrm{P} 1\left(x_{1}, y_{1}, z_{1}\right)$ and $\mathrm{P} 2\left(x_{2}, y_{2}, z_{2}\right)$ in space, the directional vector $\mathrm{Z}\left(x_{2}-x_{1} y_{2}-y_{1}, z_{2}-z_{1}\right)$ can be determined. The angle between straight line and plane can be determined by the angle for two vectors [6]. The formula can be described as

$$
\sin \varphi=\frac{|P \bullet Z|}{\|P\| \cdot\|Z\|}
$$

\subsubsection{Software Interface}

According to the method mentioned above, the attitude of the target surface can be calculated with the program. The software interface is shown in figure 3. According with the input, the attitude of the target surface can be solved directly.

At first, the input is Automatic measing points coordinate or loading points coordinate, then Set the target surface origin of coordinates in the program,The origin point and the the specified point is connected to the $\mathrm{X}$ axis, The origin point and the other the specified point is connected to the $\mathrm{Y}$ axis 
on the target surface,then the results of attitude can be obtained for calculating.the result are displayed on the interface.

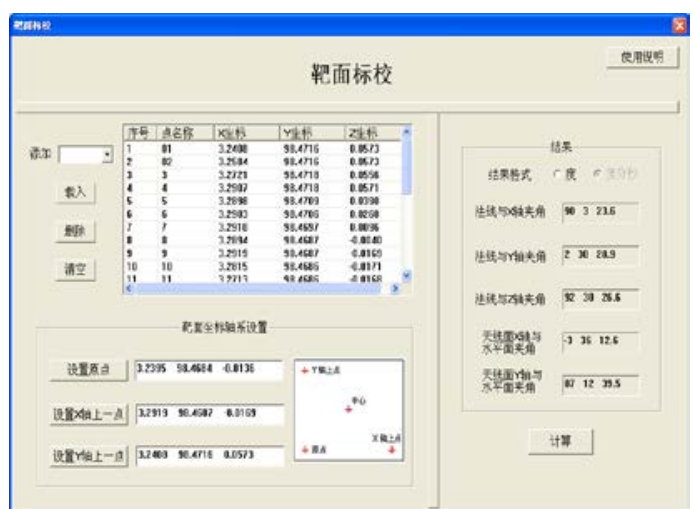

Fig3. Target plane calibration diagram

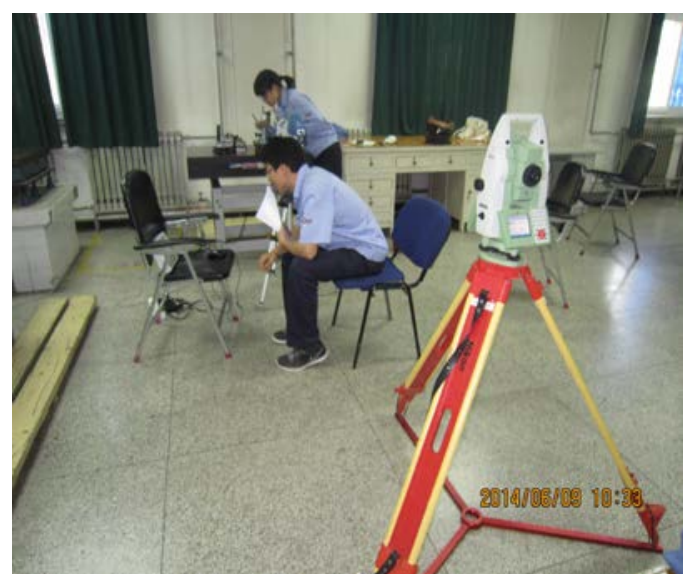

Fig4. precision measurement

\section{Test and Error Analysis}

\subsection{Checking Methods}

In order to check out the precision of this measurement method, the total station surface fitting algorithm can be accurately checked by the auto-collimation measurement algorithm. For the same mirror plane, in the same condition, by comparing the result of auto-collimation measurement algorithm and surface fitting measurement algorithm, we can see whether the results of statistical measurement accuracy is in the required range of 0.2 degrees or not. Precision detection field as shown above Figure 4.

Firstly, adjust the mirror plane and use auto-collimation measurement to obtain the angle. Then measure the points on the mirror and obtain the angles with three axes. According to the method mentioned above, measure multi groups of states and analyze whether the difference of the results, which measured by two equipments, can meet the required range or not in the same state. (the precision of auto-collimation measurement in 10 seconds)

\subsection{Test result}

Using the natations: $\Theta_{1}$ is the angle for the normal vector and $X$ axis; $\Theta_{2}$ is the angle for the normal vector and $\mathrm{Y}$ axis; $\Theta_{3}$ is the angle for the normal vector and $\mathrm{Z}$ axis.

\subsection{Test Conclusion}

Record the difference of results that measured by two kinds of measurement equipments, the difference was within 0.2 degrees. Therefore the precision of total station fitting surface completely met the requirement of the measurement precision in 0.2 degree.

\section{Conclusion}

Total station non-contact surface fitting algorithm has been used for measuring and calculating the attitude of the target surface. Through checking test, the precision of measurement has one higher order of magnitude than conventional measurement. The algorithm has been applied in the flight test of various models and obtained a good effectiveness. 
Table 1. The comparison of Auto-collimation algorithm and Plane Fitting algorithm Result

\begin{tabular}{|c|c|c|c|}
\hline No. & Auto-collimation Result & Plane Fitting Result & Difference \\
\hline \multirow{3}{*}{1} & $\Theta_{1}: 89^{\circ} 12^{\prime} 6^{\prime \prime}$ & $\Theta_{1}: 89^{\circ} 13^{\prime} 53$ & $-1^{\prime} 47^{\prime \prime}$ \\
\hline & $\Theta_{2}: 1^{\circ} 27^{\prime} 37^{\prime \prime}$ & $\Theta_{2}: 1^{\circ} 21^{\prime} 56^{\prime \prime}$ & $5^{\prime} 41^{\prime \prime}$ \\
\hline & $\Theta_{3}: 91^{\circ} 13^{\prime} 22^{\prime \prime}$ & $\Theta_{3}: 91^{\circ} 7^{\prime} 43^{\prime \prime}$ & $5^{\prime} 39^{\prime \prime}$ \\
\hline \multirow{3}{*}{2} & $\Theta_{1}: 89^{\circ} 1^{\prime} 36^{\prime \prime}$ & $\Theta_{1}: 88^{\circ} 52^{\prime} 15$ & $9^{\prime} 21^{\prime \prime}$ \\
\hline & $\Theta_{2}: 6^{\circ} 19^{\prime} 4^{\prime \prime}$ & $\Theta_{2}: 6^{\circ} 23^{\prime} 57^{\prime \prime}$ & $-4^{\prime} 53^{\prime \prime}$ \\
\hline & $\Theta_{3}: 96^{\circ} 14^{\prime} 30^{\prime \prime}$ & $\Theta_{3}: 96^{\circ} 17^{\prime} 53$ & $-3^{\prime} 23^{\prime \prime}$ \\
\hline \multirow{3}{*}{3} & $\Theta_{1}: 89^{\circ} 7^{\prime} 58^{\prime \prime}$ & $\Theta_{1}: 89^{\circ} 3^{\prime} 15^{\prime \prime}$ & $4^{\prime} 43^{\prime \prime}$ \\
\hline & $\Theta_{2}: 5^{\circ} 26^{\prime} 51^{\prime \prime}$ & $\Theta_{2}: 5^{\circ} 27^{\prime} 6^{\prime \prime}$ & $-15^{\prime \prime}$ \\
\hline & $\Theta_{3}: 95^{\circ} 22^{\prime} 39^{\prime \prime}$ & $\Theta_{3}: 95^{\circ} 22^{\prime} 6^{\prime \prime}$ & $33^{\prime \prime}$ \\
\hline \multirow{3}{*}{4} & $\Theta_{1}: 89^{\circ} 9^{\prime} 40^{\prime \prime}$ & $\Theta_{1}: 89^{\circ} 3^{\prime} 17^{\prime \prime}$ & $6^{\prime} 13^{\prime \prime}$ \\
\hline & $\Theta_{2}: 5^{\circ} 17^{\prime} 3^{\prime \prime}$ & $\Theta_{2}: 5^{\circ} 20^{\prime} 58^{\prime \prime}$ & $-3^{\prime} 55^{\prime \prime}$ \\
\hline & $\Theta_{3}: 95^{\circ} 13^{\prime} 1^{\prime \prime}$ & $\Theta_{3}: 95^{\circ} 15^{\prime} 53$ & $-2^{\prime} 52^{\prime \prime}$ \\
\hline \multirow{3}{*}{6} & $\Theta_{1}: 87^{\circ} 18^{\prime} 47^{\prime \prime}$ & $\Theta_{1}: 87^{\circ} 22^{\prime} 37$ & $-3^{\prime} 50^{\prime \prime}$ \\
\hline & $\Theta_{2}: 4^{\circ} 48^{\prime} 8^{\prime \prime}$ & $\Theta_{2}: 4^{\circ} 42^{\prime} 21^{\prime \prime}$ & $5^{\prime} 47^{\prime \prime}$ \\
\hline & $\Theta_{3}: 93^{\circ} 58^{\prime} 38^{\prime \prime}$ & $\Theta_{3}: 93^{\circ} 54^{\prime} 16$ & $4^{\prime} 22^{\prime \prime}$ \\
\hline \multirow{3}{*}{7} & $\Theta_{1}: 87^{\circ} 11^{\prime} 5^{\prime \prime}$ & $\Theta_{1}: 87^{\circ} 8^{\prime} 56^{\prime \prime}$ & $2^{\prime} 9^{\prime \prime}$ \\
\hline & $\Theta_{2}: 6^{\circ} 47^{\prime} 48^{\prime \prime}$ & $\Theta_{2}: 6^{\circ} 51^{\prime} 54^{\prime \prime}$ & $-4^{\prime} 6^{\prime \prime}$ \\
\hline & $\Theta_{3}: 96^{\circ} 10^{\prime} 52^{\prime \prime}$ & $\Theta_{3}: 96^{\circ} 14^{\prime} 23^{\prime \prime}$ & $-3^{\prime} 31^{\prime \prime}$ \\
\hline \multirow{3}{*}{8} & $\Theta_{1}: 85^{\circ} 58^{\prime} 42^{\prime \prime}$ & $\Theta_{1}: 85^{\circ} 58^{\prime} 12^{\prime \prime}$ & $30^{\prime \prime}$ \\
\hline & $\Theta_{2}: 7^{\circ} 20^{\prime} 59^{\prime \prime}$ & $\Theta_{2}: 7^{\circ} 21^{\prime} 14^{\prime \prime}$ & $-15^{\prime \prime}$ \\
\hline & $\Theta_{3}: 96^{\circ} 8^{\prime} 31^{\prime \prime}$ & $\Theta_{3}: 96^{\circ} 8^{\prime} 29^{\prime \prime}$ & $2^{\prime \prime}$ \\
\hline \multirow{3}{*}{9} & $\Theta_{1}: 91^{\circ} 32^{\prime} 54^{\prime \prime}$ & $\Theta_{1}: 91^{\circ} 34^{\prime} 30$ & $-1^{\prime} 36^{\prime \prime}$ \\
\hline & $\Theta_{2}: 6^{\circ} 18^{\prime} 24^{\prime \prime}$ & $\Theta_{2}: 6^{\circ} 12^{\prime} 50^{\prime \prime}$ & $5^{\prime} 34^{\prime \prime}$ \\
\hline & $\Theta_{3}: 96^{\circ} 6^{\prime} 43^{\prime \prime}$ & $\Theta_{3}: 96^{\circ} 0^{\prime} 34^{\prime \prime}$ & $6^{\prime} 9^{\prime \prime}$ \\
\hline
\end{tabular}

\section{References}

[1] Yang Ting-Wu,Zhang Zheng-Zhong.Optical-Electionic Measurement Theroy and Methods in Flight Test[M], Beijing:National defence ofIndustry Press, 2014,3-5

[2] Zhao Liqiang. Analysis of alignment error of dynamic reference and base for Air-to-Air Missile[J]. Aviation Weapon. 1998(4): p1-6. (in Chinese)

[3] Yang Changqi, Zhang Liehang. A Calbration Method for the Accuracy of the Air-to-Air Missile Hanging [J]. Aeronautical Science And Technology. 2009(4): p31-32. (in Chinese)

[4] Wu Rui-juan,He Hongli, Hui Guangyul,He Zhenglin.Research on Installation and Calibration of INS Base on GPS and Total Station[J].Measurement and Control Technology.2011,30(6):p8-11. (in Chinese)

[5] He Hong-li , LIU-Qian , Zhao-Jing.A High-precision Measurement for Elastic Deformation of Airplane[J]. Measurement and Control Technology.2014,33(4):p20-25. (in Chinese)

[6] Tongji University Department of Applied Mathematics. Advanced Algebra (5th edition )[M]. Higher Education Press. 2013,7. (in Chinese) 